\title{
Schlaganfall
}

\section{Prävention und Nachsorge verbessern}

Ein Drittel der Bevölkerung kennt die Risikofaktoren für den Schlaganfall nicht, berichtete Birgit Härtle, Project Manager Stroke bei Boehringer Ingelheim. Außerdem kommen 7 von $10 \mathrm{~Pa}$ tienten zu spät in die Klinik und verpassen so das Zeitfenster für eine medikamentöse Therapie. Gemäß der Devise "Zeit ist Hirn“ sei eine rasche Versorgung entscheidend, denn in jeder Minute ohne Behandlung verliere der Erkrankte zwei Millionen Nervenzellen.

\section{Erste Fortschritte durch Aufklärung}

Härtle berichtete von der Aufklärungskampagne „Herzenssache Schlaganfall“, die vom Unternehmen initiiert und in Kooperation mit der Stiftung Deutsche Schlaganfall-Hilfe sowie der Deutschen Schlaganfall-Gesellschaft umgesetzt wurde: In einem roten Doppeldecker-Bus besuchte ein Expertenteam 200 Städte und informierte etwa 60.000 Besucher über Schlaganfall-Risiken und erklärte erste Symptome sowie richtiges Handeln.

Die Kampagne zeige Erfolge: „In den Regionen, die wir besucht haben, wussten die Leute danach eher, was ein Schlaganfall ist", so Härtle. Auch Rettungsdienste wurden sensibilisiert, sodass ein größerer Anteil von Patienten innerhalb des "goldenen Zeitfensters" von $<60$ Minuten behandelt wurde. Informierte Bürger scheuten sich weniger, den Notruf zu tätigen. Bei der Anfahrt mit dem Rettungsdienst könne der Patient in der Stroke Unit angekündigt werden - dies verkürze die Zeit bis zur Therapie um 15 Minuten, was einer um $5 \%$ gesenkten Mortalität entspreche.

Analog zur Akutversorgung sollte auch die Nachsorge verbessert werden, denn auch drei Monate nach dem Schlaganfall leiden über $60 \%$ der Patienten noch unter Funktionseinschränkungen, berichtete Dr. Markus Wagner, Stiftung Deutsche Schlaganfall-Hilfe, Gütersloh. Bisher erhielten weniger als $40 \%$ aller Patienten nach der Akutbehandlung auch eine stationäre Rehabilitation.

Bei Vorhofflimmern sei die Antikoagulation die effektivste Maßnahme, um den Schlaganfall zu verhindern, sagte Prof. Joachim Röther von der Deutschen Schlaganfall-Gesellschaft. Neue orale Antikoagulanzien wie Dabigatran (Pradaxa ${ }^{\oplus}$ ) böten dabei eine bessere Prophylaxe als Vitamin-KAntagonisten.

\section{Susanne Pickl}

- Fachveranstaltung „Schlaganfall, ein verhinderbares Ereignis: Anforderungen und Notwendigkeiten im Versorgungsmanagement"; Berlin, November 2015 (Veranstalter: Boehringer Ingelheim)

\section{Asthma bronchiale}

\section{Bei der Therapie gibt es viele Hürden zu überwinden}

Eine gute Asthmakontrolle setzt ein optimales Inhalationsergebnis voraus, erklärte PD Dr. Christian Geßner, Leipzig. Um den Widerstand eines Inhalators zu überwinden und einen ausreichenden Volumenstrom zu erzeugen, ist vom $\mathrm{Pa}$ tienten eine bestimmte inspiratorische Leistung zu erbringen. Dabei stellen Dosieraerosole im Vergleich zu Pulverinhalatoren die geringeren Anforderungen an die Atemleistung. Werde dies bei der Therapiewahl berücksichtigt, sei bereits eine wichtige Hürde der Inhalationstherapie überwunden, so Geßner.

Für eine erfolgreiche Deposition des Wirkstoffs in den tiefen Atemwegen sei auch die Größe der Aerosolpartikel bzw. ein ausgewogener Feinpartikelanteil entscheidend, so Geßner. Die Fixkombination aus dem inhalativen Kortikosteroid Fluticasonpropionat und dem $\beta_{2}$-Mimetikum Formoterol im Dosieraerosol (flutiform ${ }^{\circledR}$ ) verfüge über einen günstigen
Anteil lungengängiger Feinpartikel, der mit ca. $40 \%$ konstanter als bei anderen ICS/LABA-Fixkombinationen und weitgehend unabhängig von der Flussrate sei.

\section{Fehlerhafte Bedienung des Inhalators verhindern \\ Zu den Hürden der Asthmatherapie zählt auch die fehlerhafte Bedienung des Inha- lators, so Dr. Stefan Heindl, Gauting. Die Häufigkeit kritischer Anwendungsfehler}

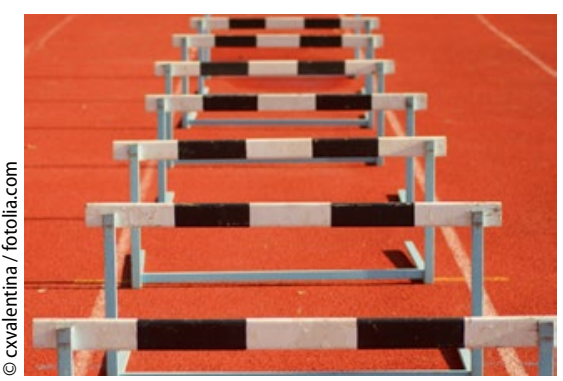

Asthmakontrolle - immer noch ein Hürdenlauf. sei bei Dosieraerosolen mit rund $12 \%$ geringer als bei Pulverinhalatoren (35-44\%)

[Melani AS et al. Respir Med. 2011].

Die effektive Wirkstoffkombination Fluticason/Formoterol weist einen sehr schnellen Wirkeintritt und eine lange Wirkdauer von 12 Stunden mit einem starken antiinflammatorischen Effekt auf. Dies wurde in der Zwischenauswertung einer nicht-interventionellen Studie zur Wirksamkeit und Sicherheit von Fluticason/Formoterol in der Praxis bestätigt. Unter der Therapie kam es zu einer klinisch relevant verbesserten Asthmakontrolle im Asthma-ControlTest $\left(\mathrm{ACT}^{\mathrm{T} \mathrm{m}}\right)$. Der Anteil der Patienten mit gut kontrolliertem Asthma hatte sich nach sechs Monaten fast verdoppelt, so Heindl.

Dagmar Jäger-Becker

- Pressegespräch „Die Hürden einer erfolgreichen Asthmatherapie und Strategien zu ihrer Überwindung"; Frankfurt, Januar 2016 (Veranstalter: Mundipharma) 